
\title{
$\begin{array}{ll}\text { Research Square } & \text { Preprints are preliminary reports that have not undergone peer review. } \\ \text { They should not be considered conclusive, used to inform clinical practice, } \\ \text { or referenced by the media as validated information. }\end{array}$
}

\section{Apolipoprotein E4 polymorphism is associated with a higher risk of aspiration pneumonia in young stroke patients}

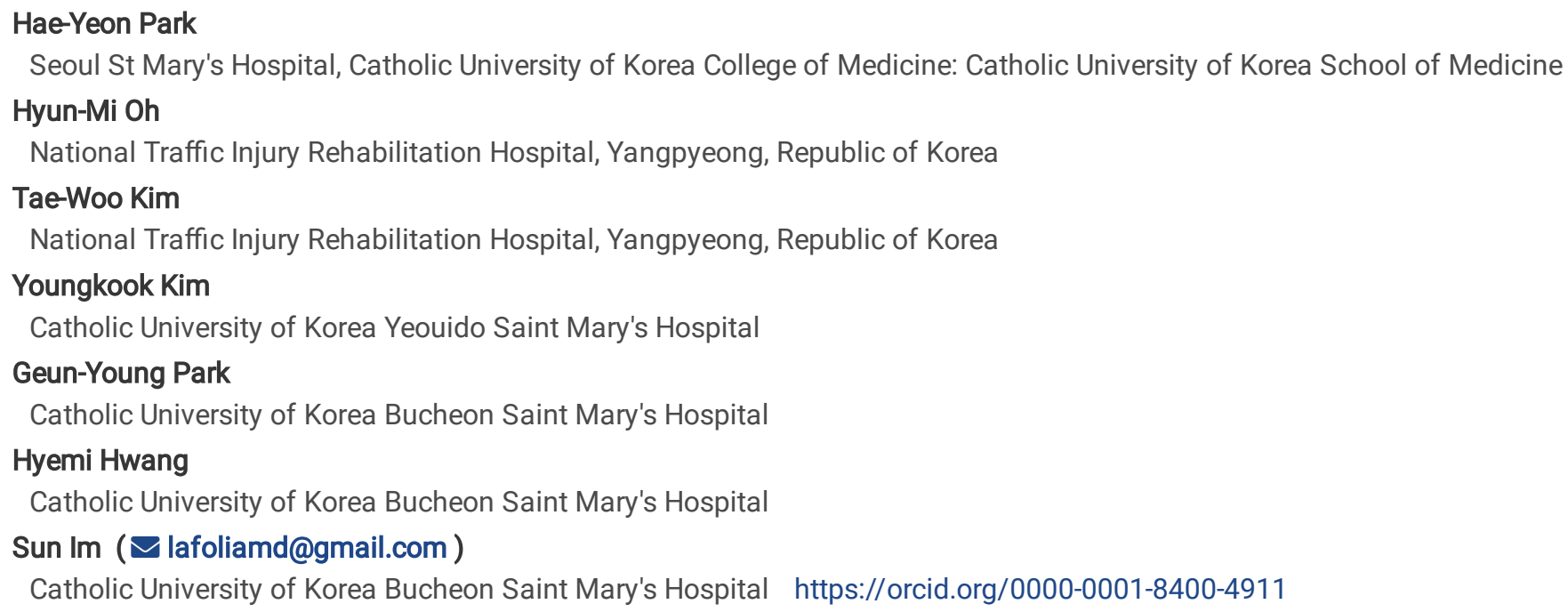

\section{Research Article}

Keywords: Deglutition, Aspiration pneumonia, Single nucleotide polymorphisms, Stroke

Posted Date: February 28th, 2022

DOI: https://doi.org/10.21203/rs.3.rs-956346/v1

License: (c) (i) This work is licensed under a Creative Commons Attribution 4.0 International License. Read Full License 


\section{Abstract}

Background Genetic polymorphisms may be associated with infections, and some genes are known to be associated with pneumonia. Aspiration pneumonia is one of the major complications after stroke, and this study aimed to evaluate genetic effects on post-stroke aspiration pneumonia.

Methods Blood samples were obtained from a total of 206 post-stroke participants (males, $n=136$; mean age, 63.8 years). Genotyping was done for catechol-0-methyltransferase (rs4680 and rs165599), dopamine receptors (DRD1; rs4532, DRD2; rs1800497, DRD3, rs6280), brain-derived neurotrophic factor (rs6265), apolipoprotein E (rs429358, rs7412), and the interleukin-1 receptor antagonist gene (rs4251961). The subjects were stratified into two groups, aged $<65$ (young) and $\geq 65$ (elderly) years. Functional parameters and swallowing outcomes were measured at enrollment and 3-months post-onset. The primary outcome was the incidence of aspiration pneumonia.

Results Analysis of the association between genetic polymorphisms and aspiration pneumonia history showed that a minor C rs 429358 allele was associated with the occurrence of aspiration pneumonia in the young group, both in the additive and dominant models (odds ratio [OR], 4.53; 95\% Confidence Interval [Cl]: $1.60-12.84, \mathrm{P}=0.004$ ). In the multivariable logistic analysis, a minor $\mathrm{C}$ rs 429358 allele increased the risk of post-stroke aspiration pneumonia by 5.35 (95\% Cl: $1.64-20.88)$. In contrast, no genetic effects were seen in the elderly group.

Conclusions A minor C rs429358 allele in young age stroke patients may increase the incidence of post-stroke aspiration pneumonia. Genetic polymorphism may provide useful information on how certain individuals are at increased risk of post-stroke aspiration pneumonia.

Clinical Trial Registration-URL: https://www.clinicaltrials.gov. Unique identifier: NCT0357744

\section{Introduction}

Aspiration pneumonia is a common medical complication after stroke, accounting for about $30-50 \%$ of the stroke population. Among the many complications, aspiration pneumonia is known as one of the leading causes of increased mortality and morbidity after stroke [1-3]. Age over 65 is an independent predictor of mortality in patients hospitalized for aspiration pneumonia [4]. Due to its detrimental effect, it is important to identify its risk factors and prevent aspiration pneumonia.

Many studies have attempted to discover the clinical factors associated with aspiration pneumonia after stroke. A prospective study of 412 patients showed that age over 65, dysarthria or aphasia, modified Rankin Scale (mRS) scores of over 4, cognitive impairment, and failure on the water swallow test were related to an increased risk of post-stroke pneumonia [5]. The brain lesion location is another important factor [3], with one recent study investigating the predictive factors of aspiration pneumonia according to different stroke lesions [6]. The levels of neurological disability and swallowing impairment are also critical predictive factors. For example, low MiniMental State Examination (MMSE) scores were significantly associated with aspiration pneumonia in supratentorial infarction, and low modified Barthel Index (MBI) scores and aspiration signs in videofluoroscopic swallowing studies (VFSSs) and salivagrams were significant predictors of infratentorial infarction. Similarly, a study of elderly stroke patients showed that multiple previous episodes of infarctions, high National Institutes of Health Stroke Scale (NIHSS) scores, and masticatory muscle paralysis were associated with poststroke aspiration pneumonia [7]. However, whether individual single nucleotide polymorphisms (SNPs) can also increase this risk and make certain individuals more vulnerable than others to respiratory complications is yet to be proven.

Recently, studies have reported certain SNPs associated with infections. For instance, increased plasma interleukin-1 receptor antagonist (IL-1ra), an endogenous immunomodulatory cytokine encoded by IL1RN, early after stroke significantly predicted post-stroke infection [8]. The minor allele of rs4252961 in IL1RN was associated with lower plasma IL-1 ra and was related to decreased risk of infections other than pneumonia $[9,10]$. In another study, apolipoprotein $\mathrm{E}$ (APOE)- $\mathbb{4}$ was found to be associated with the risk of sepsis development and progression [11]. However, to our knowledge, the genetic polymorphism influences on the development of post-stroke aspiration pneumonia, one of the most common complications after stroke, have not been well investigated yet.

Therefore, in this study, we performed a post-hoc analysis of a previous prospective trial [12] to explore whether specific genes would show an increased association of aspiration pneumonia with swallowing disturbance. In a previous trial, we assessed several SNPs and followed the patients for up to three months post-stroke. For this study, we hypothesized that specific genes may be associated with aspiration pneumonia after stroke and that the effect of these SNPs may differ according to two age groups, age $<65$ (young) and age $\geq$ 
65 (elderly) based on the previous study that age was an independent factor predicting aspiration pneumonia [4,5]. The aims of this study were first, to assess which SNP influenced aspiration pneumonia in these two age groups using a multi-inheritance model, and second, to determine whether the inclusion of this SNP with other clinical factors could increase the risk of post-stroke aspiration pneumonia.

\section{Methods Subjects}

The participants were first-ever stroke patients presenting with post-stroke dysphagia symptoms who were admitted to the department of rehabilitation medicine in two university-affiliated hospitals from August 2018 to July 2019. The inclusion criteria were patients (1) with a swallowing disorder confirmed by VFSS or fiberoptic endoscopic evaluation of swallowing (FEES), that would require modification of diet or tube feeding, (2) who had been diagnosed with a first-ever stroke, (3) who were hospitalized for 30 days and followed up three months after stroke onset, and (4) who agreed to participate in the study. The exclusion criteria were patients (1) who did not meet the inclusion criteria, (2) from whom it was difficult to collect blood for genetic testing, or (3) with long-term neurodegenerative disease such as Parkinson's disease, Alzheimer's disease, Guillain-Barre syndrome, and myasthenia gravis. The detailed description of the participants is explained in a previous study [12].

\section{Assessment Of Aspiration Pneumonia}

The primary outcome for this post-hoc study was the occurrence of aspiration pneumonia within the 3-month post-stroke period. The definition of aspiration pneumonia was (1) the presence of respiratory symptoms such as purulent sputum, tachypnea, and rales; (2) leukocytosis; (3) elevated temperature; and (4) gravitational segment infiltration on chest X-ray [13].

\section{Assessment Of Swallowing And Functional Outcomes}

Swallowing outcomes were evaluated at baseline and 3-months post-stroke. Swallowing function was evaluated using screening tools including the Gugging Swallowing Screen (GUSS) [14] and the Mann Assessment of Swallowing Ability (MASA) [15], with lower scores indicating a more severe state. The Penetration-Aspiration Scale (PAS) [16] and Modified Barium Swallow Impairment Profile (MBSImP) [17] were assessed by a videofluoroscopic swallowing study (VFSS) performed by a specialist, where the PAS indicated the presence and severity of penetration or aspiration, and $\mathrm{MBSImP}^{\odot}$ indicated impairment of the oral or pharyngeal phase. The oral intake of liquid and food was evaluated using the Functional Oral Intake Scale (FOIS) [18]. Lastly, the Eating Assessment Tool (EAT-10) questionnaire [19] was used to measure the quality of life associated with dysphagia.

Baseline stroke severity was evaluated by NIHSS scores [20]. MMSE [21] and Berg balance scale (BBS) [22] scores at baseline were assessed to evaluate baseline cognitive and balance impairment. The mRS [23], Functional Ambulatory Category (FAC) [24], and MBI [25] were assessed at baseline and 3-months post-stroke to evaluate mobility and the activities of daily living of the participants.

\section{Genotyping}

Genotyping was performed with whole blood obtained from the participants who agreed to blood sampling. The allelic discrimination of each SNP was done with $2 \mathrm{cc}$ of whole blood and TaqMan SNP Genotyping Assays. Catechol-0-methyltransferase (rs4680 and rs165599), dopamine receptor D1 (DRD1, rs4532), dopamine receptor D2 (DRD2, rs1800497), dopamine receptor D3 (DRD3, rs6280), brain-derived neurotrophic factor (rs6265), APOE (rs429358, rs7412), and the interleukin 1 receptor antagonist gene (rs4251961) were evaluated. APOE genotypes $(\varangle 2 / \varangle 2, \varangle 2 / \varangle 3, \varangle 3 / \varangle 3, \varangle 2 / \varangle 4, \varangle 3 / \varangle 4, \varangle 4 / \varangle 4)$ were further determined by allelic combinations of $r s 429358$ and rs7412 [26].

\section{Statistical Analysis}

All statistical analyses were performed using R Statistical Software (version 2.15.3; R Foundation for Statistical Computing, Vienna, Austria). The Shapiro-Wilk test for normality was used to evaluate the distribution of the continuous variables. The sample size estimation method was explained in a previous study [12]. As stated above, the enrolled participants were stratified into young ( 65 
years old) and elderly ( $\geq 65$ years old) groups. Between-group analyses were conducted using the student's t-test, Mann-Whitney test, or Chi-squared test as appropriate. To assess the association between each SNP and aspiration pneumonia, multiple inheritance models (additive, dominant, and recessive) were used. Bonferroni's correction was applied due to the evaluation of eight genes, and the significance level was determined as $\mathrm{P}<0.05 / 8=0.006$. The continuous variables are expressed as the mean and standard deviation or median with interquartile range, and the categorical variables are expressed as numbers with percentages.

Univariable and multivariable analyses were performed in each age group to evaluate the factors associated with aspiration pneumonia. The independent binary variables were rs429358 (presence of the minor allele), sex (male/female), diabetes mellitus, hypertension, alcohol, smoking, MBSImp ${ }^{\odot}$ scores (oral > 11, pharyngeal > 9, total > 22), EAT-10 (> 15), MMSE $(<18)$, and FAC (<3) scores. This study was approved by the Institutional Review Board of the Medical Center (HC17TNDI0049).

\section{Results}

\section{Participants}

A total of 218 participants were enrolled in the study, and 206 participants were available for analysis [12]. Table 1 represents the baseline characteristics of the patients according to age and history of aspiration pneumonia. In both age groups, those with a history of aspiration pneumonia had an increased risk of intubation and tracheostomy. The initial functional and swallowing outcomes were also better in those without aspiration pneumonia history, except for the MMSE scores in the elderly group and the mRS scores in both groups. In the young age group, those with aspiration pneumonia showed more chance of stroke recurrence. 
Table 1

Baseline characteristics of the participants according to age and aspiration pneumonia history

\begin{tabular}{|c|c|c|c|c|c|c|}
\hline & \multicolumn{3}{|c|}{ Young age $(n=103)$} & \multicolumn{2}{|c|}{ Old age $(n=103)$} & \multirow[b]{2}{*}{$P$ value } \\
\hline & $\mathrm{AP}(+)(n=46)$ & AP $(-)(n=57)$ & $P$ value & $\mathrm{AP}(+)(n=63)$ & AP $(-)(n=40)$ & \\
\hline Sex (male) & $31(37.4)$ & $38(66.7)$ & 1.000 & $44(69.8)$ & $23(57.5)$ & 0.285 \\
\hline BMI & $22.6 \pm 3.4$ & $23.0 \pm 2.6$ & 0.541 & $21.6 \pm 3.7$ & $22.8 \pm 3.2$ & 0.090 \\
\hline Stroke type & & & 0.366 & & & 0.093 \\
\hline infarction & 18(39.1) & $27(47.4)$ & & $40(63.5)$ & $34(85.0)$ & \\
\hline hemorrhage & $25(54.4)$ & $29(50.9)$ & & 21(33.3) & $6(15.0)$ & \\
\hline both & $3(6.5)$ & $1(1.7)$ & & $2(3.2)$ & $0(0.0)$ & \\
\hline Location & & & 0.514 & & & 0.204 \\
\hline supratentorial & $31(67.4)$ & $42(73.7)$ & & $43(68.3)$ & $33(82.5)$ & \\
\hline infratentorial & 11(23.9) & $13(22.8)$ & & 18(28.6) & $7(17.5)$ & \\
\hline multiple & $4(8.7)$ & $2(3.5)$ & & $2(3.2)$ & $0(0.0)$ & \\
\hline Side & & & 0.125 & & & 0.269 \\
\hline right & $10(21.7)$ & $23(40.4)$ & & $23(36.5)$ & $17(42.5)$ & \\
\hline left & $23(50.0)$ & $23(40.4)$ & & $28(44.4)$ & $20(50.0)$ & \\
\hline bilateral & 13(28.3) & 11(19.2) & & $12(19.0)$ & $3(7.5)$ & \\
\hline Afib & $7(15.2)$ & $3(5.3)$ & 0.173 & $16(25.4)$ & $6(15.0)$ & 0.313 \\
\hline Recur & $12(26.1)$ & $4(7.0)$ & $0.017^{\star}$ & $16(25.4)$ & $9(22.5)$ & 0.922 \\
\hline DM & $16(34.8)$ & $21(36.8)$ & 0.992 & $34(54.0)$ & $14(35.0)$ & 0.093 \\
\hline HBP & $29(63.0)$ & $40(70.2)$ & 0.579 & $52(82.5)$ & $28(70.0)$ & 0.213 \\
\hline Intubation & $27(58.7)$ & $20(35.1)$ & $0.028^{*}$ & $28(80.0)$ & $7(20.0)$ & $0.009 *$ \\
\hline Tracheostomy & 19(41.3) & $9(15.8)$ & $0.008^{*}$ & $18(100.0)$ & $0(0.0)$ & $0.001^{*}$ \\
\hline Alcohol & $14(30.4)$ & $23(40.4)$ & 0.403 & $18(28.6)$ & 13(32.5) & 0.839 \\
\hline Smoking & $14(30.4)$ & 18(31.6) & 1.000 & $18(28.6)$ & $12(30.0)$ & 1.000 \\
\hline \multicolumn{7}{|l|}{ Initial clinical outcomes } \\
\hline NIHSS & $15.2 \pm 6.6$ & $11.7 \pm 7.7$ & $0.014^{*}$ & $14.6 \pm 7.3$ & $10.1 \pm 6.2$ & $<0.001 *$ \\
\hline MMSE & $13.0[1.0-22.0]$ & $26.0[16.0-29.0]$ & $<0.001 *$ & $14.0[1.0-23.0]$ & $17.0[12.0-24.0]$ & 0.063 \\
\hline BBS & $4.0[0.0-28.0]$ & $30.0[5.0-50.0]$ & $0.001^{*}$ & $3.0[0.0-8.0]$ & $12.5[3.0-48.0]$ & $0.002^{*}$ \\
\hline NPM at $\geq 12$ weeks & $27(58.7)$ & $10(17.5)$ & $<0.001 *$ & 39 (61.9) & $4(10.0)$ & $<0.001 *$ \\
\hline MBSImp-Oral & $15.0[10.0-18.0]$ & $8.5[5.0-13.0]$ & $<0.001^{*}$ & $12.0[10.0-16.0]$ & $10.0[6.5-14.0]$ & $0.003^{*}$ \\
\hline MBSImp-Pharyngeal & $11.0[9.0-13.0]$ & $8.0[5.0-13.0]$ & $0.024 *$ & $10.0[8.0-15.0]$ & $6.0[4.5-9.0]$ & $<0.001 *$ \\
\hline GUSS & $2.0[1.0-4.0]$ & $5.0[3.0-14.0]$ & $<0.001^{*}$ & $2.0[1.0-4.0]$ & $6.0[3.0-14.0]$ & $<0.001$ * \\
\hline \multicolumn{7}{|c|}{$\begin{array}{l}\text { Values are given as number (\%), means } \pm S D \text {, or median [interquartile range]. Chi-Squared test, student t-test, or Mann-Whitney test was } \\
\text { performed to compare between groups, and P-value }<0.05 \text { are used for statistical significance. }\end{array}$} \\
\hline \multicolumn{7}{|c|}{$\begin{array}{l}\text { Abbreviations: AP, Aspiration Pneumonia; BMI, Body mass index; Afib, Atrial fibrillation; DM, Diabetes mellitus; HBP, Hypertension; } \\
\text { NIHSS, National Institutes of Health Stroke Scale; MMSE, Mini-mental state examination; BBS, berg balance scale; NPM, Nil per } \\
\text { mouth; MBSImp, Modified Barium Swallow Impairment Profile; GUSS, Gugging swallowing screen; PAS, Penetration-aspiration scale; } \\
\text { MASA, Mann Assessment of Swallowing Ability; EAT-10, Eating Assessment Tool; FOIS, Functional Oral Intake Scale; FAC, Functional } \\
\text { ambulatory category; MBI, Modified Barthel Index; mRS, modified Rankin Scale. }\end{array}$} \\
\hline
\end{tabular}




\begin{tabular}{lllllll} 
PAS & $8.0[8.0-8.0]$ & $7.0[6.0-8.0]$ & $<0.001^{*}$ & $8.0[8.0-8.0]$ & $8.0[6.0-8.0]$ & $<0.001^{*}$ \\
\hline MASA & $115.0[86.0-139.0]$ & $154.0[125.0-175.0]$ & $<0.001^{*}$ & $119.0[82.0-146.0]$ & $154.5[139.5-173.0]$ & $<0.001^{*}$ \\
\hline EAT-10 & $40.0[40.0-40.0]$ & $38.0[20.0-40.0]$ & $0.001 *$ & $40.0[40.0-40.0]$ & $32.0[20.5-40.0]$ & $<0.001^{*}$ \\
\hline FOIS & $1.0[1.0-1.0]$ & $1.0[1.0-2.0]$ & $<0.001 *$ & $1.0[1.0-1.0]$ & $1.0[1.0-2.0]$ & $0.001^{*}$ \\
FAC & $0.0[0.0-1.0]$ & $2.0[0.0-3.0]$ & $<0.001^{*}$ & $0.0[0.0-0.0]$ & $0.0[0.0-3.5]$ & $0.007 *$ \\
MBI & $8.5[0.0-44.0]$ & $53.0[23.0-82.0]$ & $<0.001 *$ & $7.0[1.5-34.0]$ & $42.5[11.5-75.5]$ & $<0.001 *$ \\
mRS $(\geq 3)$ & $46(100)$ & $55(96.5)$ & 0.572 & $61(96.8)$ & $38(95.0)$ & 1.000
\end{tabular}

Values are given as number (\%), means $\pm S D$, or median [interquartile range]. Chi-Squared test, student t-test, or Mann-Whitney test was performed to compare between groups, and P-value $<0.05$ are used for statistical significance.

Abbreviations: AP, Aspiration Pneumonia; BMI, Body mass index; Afib, Atrial fibrillation; DM, Diabetes mellitus; HBP, Hypertension; NIHSS, National Institutes of Health Stroke Scale; MMSE, Mini-mental state examination; BBS, berg balance scale; NPM, Nil per mouth; MBSImp, Modified Barium Swallow Impairment Profile; GUSS, Gugging swallowing screen; PAS, Penetration-aspiration scale; MASA, Mann Assessment of Swallowing Ability; EAT-10, Eating Assessment Tool; FOIS, Functional Oral Intake Scale; FAC, Functional ambulatory category; MBI, Modified Barthel Index; mRS, modified Rankin Scale.

When only participants with a history of aspiration pneumonia were evaluated, there was a higher proportion of infarction and hypertension in the elderly, compared to the young group (data not shown).

\section{Genetic Polymorphism And Association With Aspiration Pneumonia}

The analysis of the association between SNP polymorphisms and aspiration pneumonia history is shown in Table 2. Three gene models (additive, dominant, and recessive) showed that the minor C rs429358 allele was associated with the occurrence of aspiration pneumonia in the young group, both in the additive and dominant models (Odds Ratio [OR] = 4.53; 95\% Confidence Interval [CI]: 1.60 $12.84, \mathrm{P}=0.004)$. However, no such association was seen in the elderly group. 
Table 2

Analysis of APOE4 (rs429358) polymorphisms and statistical association with aspiration pneumonia

\begin{tabular}{|c|c|c|c|c|c|c|c|c|c|}
\hline & \multicolumn{3}{|c|}{ All Ages $(n=206)$} & \multicolumn{3}{|c|}{ Age $<65(n=103)$} & \multicolumn{3}{|c|}{ Age $\geq 65(n=103)$} \\
\hline & Additive & Dominant & Recessive & Additive & Dominant & Recessive & Additive & Dominant & Recessive \\
\hline & $\begin{array}{l}\text { OR } \\
(95 \% \mathrm{Cl})\end{array}$ & $\begin{array}{l}\text { OR } \\
(95 \% \mathrm{Cl})\end{array}$ & $\begin{array}{l}\text { OR } \\
(95 \% \mathrm{Cl})\end{array}$ & $\begin{array}{l}\text { OR } \\
(95 \% \mathrm{Cl})\end{array}$ & $\begin{array}{l}\text { OR } \\
(95 \% \mathrm{Cl})\end{array}$ & $\begin{array}{l}\text { OR } \\
(95 \% \mathrm{Cl})\end{array}$ & $\begin{array}{l}\text { OR } \\
(95 \% \mathrm{Cl})\end{array}$ & $\begin{array}{l}\text { OR } \\
(95 \% \mathrm{Cl})\end{array}$ & $\begin{array}{l}\text { OR } \\
(95 \% \mathrm{Cl})\end{array}$ \\
\hline \multirow[t]{2}{*}{ rs429358 } & 1.92 & 1.87 & $\mathrm{~N} / \mathrm{A}$ & 4.53 & 4.53 & $\mathrm{~N} / \mathrm{A}$ & 0.98 & 0.82 & N/A \\
\hline & $\begin{array}{l}(1.01- \\
3.65)^{\star}\end{array}$ & $\begin{array}{l}(0.96- \\
3.65)\end{array}$ & & $\begin{array}{l}(1.60- \\
12.84)^{\dagger}\end{array}$ & $\begin{array}{l}(1.60- \\
12.84)^{\dagger}\end{array}$ & & $\begin{array}{l}(0.43- \\
2.21)\end{array}$ & $\begin{array}{l}(0.33- \\
2.31)\end{array}$ & \\
\hline \multirow[t]{2}{*}{ rs7412 } & 0.73 & 0.73 & $\mathrm{~N} / \mathrm{A}$ & 0.99 & 0.99 & $\mathrm{~N} / \mathrm{A}$ & 0.5 & 0.5 & $\mathrm{~N} / \mathrm{A}$ \\
\hline & $\begin{array}{l}(0.31- \\
1.7)\end{array}$ & $(0.31-1.7)$ & & $\begin{array}{l}(0.25- \\
3.92)\end{array}$ & $\begin{array}{l}(0.25- \\
3.92)\end{array}$ & & $\begin{array}{l}(0.17- \\
1.51)\end{array}$ & $\begin{array}{l}(0.17- \\
1.51)\end{array}$ & \\
\hline \multirow[t]{2}{*}{ rs165599 } & 1.20 & 1.30 & 1.23 & 1.18 & 1.59 & 0.88 & 1.28 & 1.07 & 2.12 \\
\hline & $\begin{array}{l}(0.80- \\
1.82)\end{array}$ & $\begin{array}{l}(0.71- \\
2.37)\end{array}$ & $\begin{array}{l}(0.59- \\
2.56)\end{array}$ & $\begin{array}{l}(0.66- \\
2.08)\end{array}$ & $\begin{array}{l}(0.66- \\
3.81)\end{array}$ & $\begin{array}{l}(0.32- \\
2.41)\end{array}$ & $\begin{array}{l}(0.69- \\
2.36)\end{array}$ & $\begin{array}{l}(0.45- \\
2.56)\end{array}$ & $\begin{array}{l}(0.63- \\
7.10)\end{array}$ \\
\hline \multirow[t]{2}{*}{ rs4251961 } & 0.95 & 0.95 & 0.89 & 0.78 & 0.84 & $\mathrm{~N} / \mathrm{A}$ & 1.42 & 1.31 & $\mathrm{~N} / \mathrm{A}$ \\
\hline & $\begin{array}{l}(0.47- \\
1.93)\end{array}$ & $\begin{array}{l}(0.43- \\
2.08)\end{array}$ & $\begin{array}{l}(0.06- \\
14.40)\end{array}$ & $\begin{array}{l}(0.29- \\
2.07)\end{array}$ & $\begin{array}{l}(0.29- \\
2.42)\end{array}$ & & $\begin{array}{l}(0.44- \\
4.55)\end{array}$ & $\begin{array}{l}(0.37- \\
4.67)\end{array}$ & \\
\hline \multirow[t]{2}{*}{ rs4532 } & 0.92 & 0.92 & 0.89 & 1.17 & 1.32 & $\mathrm{~N} / \mathrm{A}$ & 0.77 & 0.65 & 1.28 \\
\hline & $\begin{array}{l}(0.51- \\
1.67)\end{array}$ & $\begin{array}{l}(0.47- \\
1.79)\end{array}$ & $\begin{array}{l}(0.12- \\
6.43)\end{array}$ & $\begin{array}{l}(0.50- \\
2.77)\end{array}$ & $\begin{array}{l}(0.53- \\
3.31)\end{array}$ & & $\begin{array}{l}(0.34- \\
1.75)\end{array}$ & $\begin{array}{l}(0.24- \\
1.77)\end{array}$ & $\begin{array}{l}(0.11- \\
14.58)\end{array}$ \\
\hline \multirow[t]{2}{*}{ rs1800497 } & 1.06 & 1.06 & 1.11 & 1.59 & 1.53 & 2.36 & 0.77 & 0.85 & 0.50 \\
\hline & $\begin{array}{l}(0.70- \\
1.59)\end{array}$ & $\begin{array}{l}(0.60- \\
1.87)\end{array}$ & $\begin{array}{l}(0.51- \\
2.45)\end{array}$ & $\begin{array}{l}(0.88- \\
2.89)\end{array}$ & $\begin{array}{l}(0.65- \\
3.60)\end{array}$ & $\begin{array}{l}(0.79- \\
7.08)\end{array}$ & $\begin{array}{l}(0.43- \\
1.39)\end{array}$ & $\begin{array}{l}(0.38- \\
1.93)\end{array}$ & $\begin{array}{l}(0.15- \\
1.60)\end{array}$ \\
\hline \multirow[t]{2}{*}{ rs6280 } & 1.03 & 1.11 & 0.73 & 1.18 & 1.52 & 0.29 & 0.84 & 0.73 & 1.29 \\
\hline & $\begin{array}{l}(0.65- \\
1.64)\end{array}$ & $\begin{array}{l}(0.64- \\
1.93)\end{array}$ & $\begin{array}{l}(0.22- \\
2.47)\end{array}$ & $\begin{array}{l}(0.61- \\
2.28)\end{array}$ & $\begin{array}{l}(0.70- \\
3.33)\end{array}$ & $\begin{array}{l}(0.03- \\
2.73)\end{array}$ & $\begin{array}{l}(0.43- \\
1.63)\end{array}$ & $\begin{array}{l}(0.33- \\
1.64)\end{array}$ & $\begin{array}{l}(0.22- \\
7.38)\end{array}$ \\
\hline \multirow[t]{2}{*}{ rs4680 } & 0.88 & 0.88 & 0.75 & 0.84 & 0.73 & 0.99 & 0.86 & 0.88 & 0.62 \\
\hline & $\begin{array}{l}(0.56- \\
1.37)\end{array}$ & $\begin{array}{l}(0.51- \\
1.52)\end{array}$ & $\begin{array}{l}(0.24- \\
2.31)\end{array}$ & $\begin{array}{l}(0.46- \\
1.53)\end{array}$ & $\begin{array}{l}(0.33- \\
1.64)\end{array}$ & $\begin{array}{l}(0.25- \\
3.92)\end{array}$ & $\begin{array}{l}(0.43- \\
1.71)\end{array}$ & $\begin{array}{l}(0.40- \\
1.94)\end{array}$ & $\begin{array}{l}(0.08- \\
4.61)\end{array}$ \\
\hline \multirow[t]{2}{*}{ rs6265 } & 1.14 & 1.04 & 1.44 & 1.23 & 1.20 & 1.48 & 1.14 & 1.04 & 1.48 \\
\hline & $\begin{array}{l}(0.80- \\
1.64)\end{array}$ & $\begin{array}{l}(0.58- \\
1.87)\end{array}$ & $\begin{array}{l}(0.76- \\
2.72)\end{array}$ & $\begin{array}{l}(0.73- \\
2.10)\end{array}$ & $\begin{array}{l}(0.51- \\
2.87)\end{array}$ & $\begin{array}{l}(0.61- \\
3.58)\end{array}$ & $\begin{array}{l}(0.68- \\
1.92)\end{array}$ & $\begin{array}{l}(0.46- \\
2.37)\end{array}$ & $\begin{array}{l}(0.57- \\
3.84)\end{array}$ \\
\hline \multicolumn{10}{|c|}{ Major allele; M, Minor allele; m } \\
\hline \multicolumn{10}{|c|}{ Additive; MM (Reference) vs Mm + 2*mm (assumption: dose-response, $\mathrm{MM}<\mathrm{Mm}<\mathrm{mm}$ ) } \\
\hline \multicolumn{10}{|c|}{ Dominant; MM (Refernece) vs Mm + mm } \\
\hline \multicolumn{10}{|c|}{ Recessive; MM + Mm (Reference) vs mm } \\
\hline \multicolumn{10}{|c|}{${ }^{*} \mathrm{P}$ value $=0.046 ;{ }^{\dagger} \mathrm{P}$-value $=0.004$} \\
\hline
\end{tabular}

\section{Apoe Genotyping}

APOE genotyping $(\varangle 2 / \varangle 2, \varangle 2 / \varangle 3, \varangle 3 / \varangle 3, \varangle 2 / \varangle 4, \varangle 3 / \varangle 4, \varangle 4 / \varangle 4)$ was performed by the combination of $r s 429358$ and $r s 7412$ [26]. No participants had the APOE $₫ 2 / \varangle 2$ genotype in this study. Those with the minor C rs 429358 allele (either CT or CC) were categorized as 


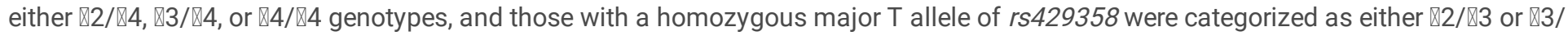
$\varangle 3$ genotypes, regardless of the $r s 7412$ allele. Therefore, those with a minor C rs 429358 allele were categorized as APOE $₫ 4$.

\section{Comparison By Apoe Genotypes}

The comparison of baseline parameters according to APOE genotype in the young age group showed that those with APOE $₫ 4$ had more incidence of aspiration pneumonia and showed poorer initial PAS scores (Supplementary Table 1). In contrast, the proportion of patients with post-stroke aspiration pneumonia was significantly higher in those with APOE $₫ 4$ in the young age group, and the incidence of urinary tract infections and colitis did not differ according to APOE genotypes (Figure 1). In the elderly group, no baseline functional and swallowing parameters significantly differed according to the presence of APOE $₫ 4$ (Supplementary Table 2).

The evaluation of changes in the swallowing and functional parameters from baseline to 3 months showed no statistically significant differences according to APOE genotypes, both in the young and elderly groups (Supplementary Table 2).

\section{Univariable And Multivariable Analysis Of Predictors Of Aspiration Pneumonia}

The minor C rs429358 allele (OR = 5.35; 95\% Cl: 1.64 - 20.88), poor oral MBSImP ${ }^{\odot}$ stage score (OR = 2.73; $95 \%$ Cl: $\left.1.04-7.41\right)$, and higher $\mathrm{mRS}$ scores $(\mathrm{OR}=2.45 ; 95 \% \mathrm{Cl}: 1.30-5.00)$ were associated with an increased occurrence of aspiration pneumonia in the young age group. In this group, a final multivariable model that included $r s 429358$, poor oral MBSImP ${ }^{\odot}$ stage score, worse PAS scores, and higher mRS scores, showed an area under the receiver operating characteristic curve (AUROC) of 0.82 (95\% Cl: $0.74-0.90)$ (Table 3).

Table 3

Univariable and Multivariable analysis in Young Age

\begin{tabular}{|c|c|c|c|c|}
\hline & Univariate & & Multivariate & \\
\hline & OR $(95 \% \mathrm{Cl})$ & P-value & OR $(95 \% \mathrm{Cl})$ & P-value \\
\hline rs429358 & $4.53(1.67-13.80)$ & $0.004^{\star}$ & $5.35(1.64-20.88)$ & $0.009 *$ \\
\hline Sex (male) & $0.97(0.42-2.21)$ & 0.938 & & \\
\hline BMI & $0.98(0.85-1.11)$ & 0.722 & & \\
\hline DM (yes) & $0.91(0.40-2.06)$ & 0.829 & & \\
\hline HBP & $0.73(0.32-1.66)$ & 0.445 & & \\
\hline Alcohol (yes) & $0.65(0.28-1.46)$ & 0.298 & & \\
\hline Smoking (yes) & $0.95(0.41-2.19)$ & 0.901 & & \\
\hline MBSImp-Oral (>11) & $4.03(1.79-9.43)$ & $0.001^{*}$ & $2.73(1.04-7.41)$ & $0.043^{*}$ \\
\hline PAS & $1.92(1.31-3.24)$ & $0.004^{\star}$ & $1.42(0.97-2.43)$ & 0.126 \\
\hline EAT-10 (>15) & $6.30(1.06-120.15)$ & 0.091 & & \\
\hline $\mathrm{mRS}$ & $2.77(1.63-5.02)$ & $<0.001^{*}$ & $2.45(1.30-5.00)$ & $0.009 *$ \\
\hline AUROC & & & $0.82(0.74-0.90)$ & \\
\hline \multicolumn{5}{|c|}{$\begin{array}{l}\text { Independent binary variables are rs } 429358 \text { (presence of minor allele), sex (male/female), diabetes mellitus, hypertension, alcohol, } \\
\text { smoking, MBSImp scores (oral>11), and EAT-10 (>15) }\end{array}$} \\
\hline \multicolumn{5}{|c|}{ P-value $<0.05$ are used for statistical significance. } \\
\hline
\end{tabular}

In contrast, in the elderly group, rs 429358 did not significantly increase the risk of aspiration pneumonia. Instead, among the included factors, poor GUSS scores ( $\mathrm{OR}=0.80 ; 95 \% \mathrm{Cl}$ : $0.68-0.93)$ and PAS scores $(\mathrm{OR}=1.88 ; 95 \% \mathrm{Cl}: 1.22-3.39)$ increased the aspiration pneumonia risk in a multivariable model with an AUROC of 0.89 (95\% Cl: $0.83-0.95)$ (Table 4). 
Univariable and Multivariable analysis in Old Age

\begin{tabular}{|c|c|c|c|c|}
\hline & \multicolumn{2}{|l|}{ Univariate } & \multicolumn{2}{|l|}{ Multivariate } \\
\hline & OR (95\% Cl) & $p$-value & OR (95\% Cl) & P-value \\
\hline rs429358 & $0.82(0.33-2.07)$ & 0.667 & & \\
\hline Sex (male) & $0.64(0.27-1.49)$ & 0.295 & & \\
\hline BMI & $0.91(0.81-1.02)$ & 0.114 & & \\
\hline DM (yes) & $2.10(0.92-4.90)$ & 0.082 & & \\
\hline HBP (yes) & $1.82(0.69-4.79)$ & 0.223 & & \\
\hline NIHSS & $1.10(1.03-1.17)$ & $0.005^{\star}$ & $1.06(0.97-1.16)$ & 0.188 \\
\hline Alcohol (yes) & $0.86(0.35-2.11)$ & 0.732 & & \\
\hline Smoking (yes) & $0.89(0.36-2.25)$ & 0.806 & & \\
\hline MMSE (<18) & $1.10(0.49-2.49)$ & 0.819 & & \\
\hline MBSImp-Oral (>11) & $2.35(1.03-5.57)$ & $0.047^{\star}$ & $0.26(0.06-0.98)$ & 0.062 \\
\hline GUSS & $0.78(0.69-0.86)$ & $<0.001^{*}$ & $0.80(0.68-0.93)$ & $0.007 *$ \\
\hline PAS & $2.03(1.36-3.38)$ & $0.002^{\star}$ & $1.88(1.22-3.39)$ & $0.013^{*}$ \\
\hline EAT-10 (>15) & $5.06(0.62-104.41)$ & 0.168 & & \\
\hline FOIS & $0.31(0.11-0.61)$ & $0.006^{*}$ & $0.43(0.12-1.19)$ & 0.150 \\
\hline FAC $(<3)$ & $6.42(2.19-21.74)$ & $0.001 *$ & $4.12(0.94-19.96)$ & 0.064 \\
\hline AUROC & & & $0.89(0.83-0.95)$ & \\
\hline \multicolumn{5}{|c|}{$\begin{array}{l}\text { Independent binary variables are rs429358 (presence of minor allele), sex (male/female), diabetes mellitus, hypertension, alcohol, } \\
\text { smoking, MMSE (<18), MBSImp scores (oral>11), EAT-10 (>15), and FAC }(<3)\end{array}$} \\
\hline \multicolumn{5}{|c|}{ P-value $<0.05$ are used for statistical significance } \\
\hline \multicolumn{5}{|c|}{$\begin{array}{l}\text { Abbreviations: OR, Odds ratio; Cl, Confidence interval; BMI, Body mass index; DM, Diabetes mellitus; HBP, Hypertension; NIHSS, } \\
\text { National Institutes of Health Stroke Scale; MMSE, Mini-mental state examination; MBSImp, Modified Barium Swallow Impairment } \\
\text { Profile; GUSS, Gugging swallowing screen; PAS, Penetration-aspiration scale; EAT-10, Eating Assessment Tool; FOIS, Functional Oral } \\
\text { Intake Scale; FAC, Functional ambulatory category; AUROC, Area under receiver operating characteristic curve. }\end{array}$} \\
\hline
\end{tabular}

\section{Discussions}

This study showed that the predictive factors for aspiration pneumonia may differ between young and elderly stroke patients, with SNP influences manifested mainly in the former group. Among the various SNPs analyzed, only the APOE genotypes showed a positive association with the minor C rs 429358 allele, increasing the risk of aspiration pneumonia in young post-stroke dysphagia patients. With the inclusion of this SNP, a multivariable model that included worse baseline $\mathrm{mRS}$ and $\mathrm{MBSImP}{ }^{\odot}$ scores predicted the risk of aspiration pneumonia in the young group with high accuracy (AUROC $=0.82(95 \% \mathrm{Cl}: 0.74-0.90)$ ). In contrast, in the elderly group, a model that included only the clinical factors with worse initial GUSS and PAS scores was shown to predict the risk of aspiration pneumonia (AUROC $=0.89(95 \% \mathrm{Cl}: 0.83-0.95))$.

The well-known factors that can increase the risk of aspiration pneumonia after stroke are increased age, a higher degree of cognitive impairment, low levels of substance $\mathrm{P}$ or the use of angiotensin-converting enzyme inhibitors, increased severity of aspiration and dysphagia, and increased levels of post-stroke disability [5-7, 27-29]. Our study's results were consistent with those of previous studies, reflected by the lower GUSS and higher PAS and NIHSS scores in the elderly group and the increased severity of the oral stage of impairment in the younger age stroke group with higher initial mRS scores. Of significant interest was that in the young age group, the multiple inheritance analysis showed that certain SNPs, specifically rs4252961, were also associated with an increased risk of aspiration pneumonia. 
One of the unexpected findings was that APOE was the SNP associated with aspiration pneumonia, which is discrepant with previous findings that reported that the major allele of rs4252961 in IL1RN was associated with infection risk [8]. APOE is encoded by three alleles $(\varangle 2, \varangle 3$, and $\varangle 4)$ on chromosome $19 q 13$, and is produced by the liver, brain, spleen, kidneys, lungs, and muscle tissue [30]. By binding to lipoprotein particles and cellular receptors, APOE controls triglyceride homeostasis and lipoprotein clearance in tissues including the lungs [31]. This regulation is affected by APOE polymorphism, and APOE $₫ 4$ polymorphism is known to increase the risk of atherosclerosis and neurodegenerative disorders, including Alzheimer's dementia. This allele can also lead to higher mortality in dementia patients and is known to be associated with younger stroke onset [32-35].

Previous studies have discussed the role of APOE genotypes in infection especially in respiratory disease. APOE acts on macrophages and $T$ cells, regulating the innate immune system. In one study, when injected with bacterial endotoxin, those with APOE $\triangle 4$ showed higher hyperthermia and plasma tumor necrosis factor-alpha (TNF-a) levels [36]. APOE $₫ 4$ has also been shown to be related to the development of sepsis [11], and enhanced the attachment of Chlamydia pneumonia elementary bodies to host cells [37]. Furthermore, in a recent study, those with APOE $₫ 4$ showed a higher risk of severe COVID-19 infections [38].

Another point of interest from our study was that SNP showed different effects in young versus elderly stroke patients. Only young adults with a minor C rs429358 allele showed an increased risk of aspiration pneumonia in multiple inheritance models, including both additive and dominant models, showing that APOE $₫ 4$ increased the risk of aspiration pneumonia with an OR of 4.53 , even after multiple Bonferroni corrections. When included in the multivariable analysis model, this minor C rs429358 allele remained an important risk factor for aspiration pneumonia. However, the effects of this SNP on infection were only observed for aspiration pneumonia. Minor APOE alleles seemed not to exert a similar influence on urinary or gastrointestinal tract infections. The predilection of APOE for pneumonia is consistent with past studies advocating that the role of APOE $₫ 4$ in lung disease may be linked to the increased risk of pulmonary complications [37, 38].

In contrast, no APOE allele seemed to play a role in increasing the risk of aspiration pneumonia in the elderly group. Since old age itself is a vital risk factor for aspiration pneumonia [4,5], genetic polymorphism influences may have been overridden by this age factor and thus, be less influenced when compared to the young age group. In fact, $44.6 \%$ of the young and $61.2 \%$ of the elderly patients had aspiration pneumonia history, with more aspiration pneumonia in the elderly group regardless of APOE $₫ 4$ presence.

Some limitations of the study need to be considered. First, in this study, we compared the groups based on the presence of APOE $₫ 4$,

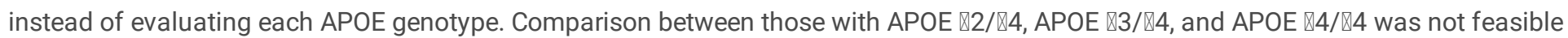

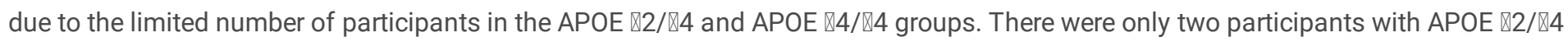

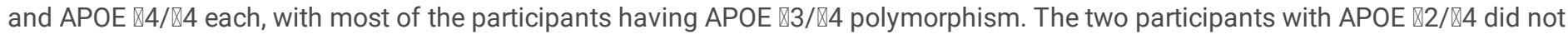
experience aspiration pneumonia, whereas two APOE $₫ 4 / \varangle 4$ participants had aspiration pneumonia during the follow-up period, which may support our results that APOE $\mathbb{4}$ may act as a risk factor for aspiration pneumonia after stroke. Secondly, recruitment was done in only two university-affiliated hospitals, leading to possible selection bias. Also, the study was conducted only on post-stroke patients. Therefore, the results of the study may not be generalizable to all patients with aspiration pneumonia related to deglutition impairment caused by other neurodegenerative disorders. In the future, a multi-center prospective study with a larger number of participants of each genotype with different etiologies is needed to further support our findings.

Nevertheless, the results of this study are noteworthy since according to our knowledge, this is one of the first studies to explore the effects of genetic polymorphisms on post-stroke aspiration pneumonia. Many studies have attempted to identify factors based on clinical information $[39,40]$, and neuroanatomical correlates $[41,42]$ to help determine those at an increased risk of aspiration pneumonia. Our results may provide information on the complexities of understanding how the immune responds following stroke and how certain SNPs may modulate the risk of infection.

In summary, this study suggests that APOE $₫ 4$ may be a risk factor for post-stroke aspiration pneumonia in patients younger than 65 years old. Considering that aspiration pneumonia may increase post-stroke mortality, this new role of APOE may be clinically relevant. Genetic polymorphisms may be taken into consideration in predicting post-stroke aspiration pneumonia.

\section{Declarations}

\section{Acknowledgments}

The statistical consultation was supported by the Department of Biostatistics of the Catholic Research Coordinating Center. 


\section{Funding}

This work was supported by the National Research Foundation of Korea (NRF) grant funded by the Korea government (MSIT) 2017R1C1B501792.

\section{Conflicts of interest}

The authors declare that there is no conflict of interest.

\section{Availability of data and material}

The data that support the findings of this study are available on request from the corresponding author. The data are not publicly available due to ethical restrictions.

\section{Code availability}

Statistical R-studio codes can be made available upon reasonable request.

\section{Authors' contributions}

G.P and S.I. were responsible for conceptualization, methodology, and study administration; S.I. performed data curation and analysis; H.P. and S.I. wrote the original draft, which was revised and edited by H.H, H.O., T.K., and Y.K.

\section{Ethical approval}

The study was approved by the institutional review board of Catholic Medical Center (HC17TNDI0049).

\section{Consent to participate}

This study was undertaken with written informed consent from all patients or legal guardians for genetic testing

\section{Consent for publication}

All authors agreed to the publication of the manuscript in its current form.

\section{References}

1. Armstrong JR, Mosher BD. Aspiration pneumonia after stroke: intervention and prevention. Neurohospitalist. 2011;1:85-93. https://doi.org/10.1177/1941875210395775.

2. Feng MC, Lin YC, Chang YH, Chen $\mathrm{CH}$, Chiang HC, Huang LC, Yang YH, Hung CH. The Mortality and the Risk of Aspiration Pneumonia Related with Dysphagia in Stroke Patients. J Stroke Cerebrovasc Dis. 2019;28:1381-7. https://doi.org/10.1016/j.jstrokecerebrovasdis.2019.02.011.

3. Roje-Bedeković M, Dimitrović A, Breitenfeld T, Supanc V, Vargek Solter V. Reliable predicting factors for post-stroke dysphagia - Our experience. Neurology Psychiatry Brain Research. 2020;38:97-101. https://doi.org/https://doi.org/10.1016/j.npbr.2020.10.006.

4. Wu CP, Chen YW, Wang MJ, Pinelis E. National Trends in Admission for Aspiration Pneumonia in the United States, 2002-2012. Ann Am Thorac Soc. 2017;14:874-9. https://doi.org/10.1513/AnnalsATS.201611-8670C.

5. Sellars C, Bowie L, Bagg J, Sweeney MP, Miller H, Tilston J, Langhorne P, Stott DJ. Risk factors for chest infection in acute stroke: a prospective cohort study. Stroke. 2007;38:2284-91. https://doi.org/10.1161/STROKEAHA.106.478156.

6. Yu KJ, Moon H, Park D. Different clinical predictors of aspiration pneumonia in dysphagic stroke patients related to stroke lesion: A STROBE-complaint retrospective study. Med (Baltim). 2018;97:e13968. https://doi.org/10.1097/MD.0000000000013968.

7. Xu Z, Gu Y, Li J, Wang C, Wang R, Huang Y, Zhang J. Dysphagia and aspiration pneumonia in elderly hospitalization stroke patients: Risk factors, cerebral infarction area comparison. J Back Musculoskelet Rehabil. 2019;32:85-91. https://doi.org/10.3233/BMR170801.

8. Tanzi P, Cain K, Kalil A, Zierath D, Savos A, Gee JM, Shibata D, Hadwin J, Carter K, Becker K. Post-stroke infection: a role for IL-1ra? Neurocrit Care. 2011;14:244-52. https://doi.org/10.1007/s12028-010-9490-7. 
9. Rafiq S, Stevens K, Hurst AJ, Murray A, Henley W, Weedon MN, Bandinelli S, Corsi AM, Guralnik JM, Ferruci L, Melzer D, Frayling TM. Common genetic variation in the gene encoding interleukin-1-receptor antagonist (IL-1RA) is associated with altered circulating IL1RA levels. Genes Immun. 2007;8:344-51. https://doi.org/10.1038/sj.gene.6364393.

10. Becker KJ, Dankwa D, Lee R, Schulze J, Zierath D, Tanzi P, Cain K, Dressel A, Shibata D, Weinstein J. Stroke, IL-1 ra, IL1RN, infection and outcome. Neurocrit Care. 2014;21:140-6. https://doi.org/10.1007/s12028-013-9899-x.

11. Shao Y, Zhao T, Zhang W, He J, Lu F, Cai Y, Lai Z, Wei N, Liang C, Liu L, Hong Y, Cheng X, Li J, Tang P, Fan W, Ou M, Yang J, Liu Y, Cui L. Presence of the apolipoprotein E-epsilon4 allele is associated with an increased risk of sepsis progression. Sci Rep. 2020;10:15735. https://doi.org/10.1038/s41598-020-72616-0.

12. Oh HM, Kim TW, Park HY, Kim Y, Park GY, Im S. Role of rs6265 BDNF polymorphisms and post-stroke dysphagia recovery-A prospective cohort study. Neurogastroenterol Motil. 2021;33:e13953. https://doi.org/10.1111/nmo.13953.

13. Martin BJ, Corlew MM, Wood H, Olson D, Golopol LA, Wingo M, Kirmani N. The association of swallowing dysfunction and aspiration pneumonia. Dysphagia. 1994;9:1-6. https://doi.org/10.1007/BF00262751.

14. John JS, Berger L. Using the gugging swallowing screen (GUSS) for dysphagia screening in acute stroke patients. J Contin Educ Nurs. 2015;46:103-4. https://doi.org/10.3928/00220124-20150220-12.

15. Oh J-C. (2014) Reliability and validity of Korean Mann assessment of swallowing ability(K-MASA). Dissertation, Graduate School, Yonsei University.

16. Rosenbek JC, Robbins JA, Roecker EB, Coyle JL, Wood JL. A penetration-aspiration scale. Dysphagia. 1996;11:93-8. https://doi.org/10.1007/bf00417897.

17. Martin-Harris B, Brodsky MB, Michel Y, Castell DO, Schleicher M, Sandidge J, Maxwell R, Blair J. MBS measurement tool for swallow impairment-MBSImp: establishing a standard. Dysphagia. 2008;23:392-405. https://doi.org/10.1007/s00455-008-9185-9.

18. Crary MA, Mann GD, Groher ME. Initial psychometric assessment of a functional oral intake scale for dysphagia in stroke patients. Arch Phys Med Rehabil. 2005;86:1516-20. https://doi.org/10.1016/j.apmr.2004.11.049.

19. Belafsky PC, Mouadeb DA, Rees CJ, Pryor JC, Postma GN, Allen J, Leonard RJ. Validity and reliability of the Eating Assessment Tool (EAT-10). Ann Otol Rhinol Laryngol. 2008;117:919-24. https://doi.org/10.1177/000348940811701210.

20. Goldstein LB, Samsa GP. Reliability of the National Institutes of Health Stroke Scale. Extension to non-neurologists in the context of a clinical trial. Stroke. 1997;28:307-10.

21. Park J-H, Kwon YC. Modification of the mini-mental state examination for use in the elderly in a non-western society. Part 1. Development of korean version of mini-mental state examination. Int J Geriatr Psychiatry. 1990;5:381-7. https://doi.org/10.1002/gps.930050606.

22. Berg K, Wood-Dauphine S, Williams J, Gayton DJPC. Measuring balance in the elderly: preliminary development of an instrument. Physiother Can. 1989;41:304-11.

23. Burn JP. Reliability of the modified Rankin Scale. Stroke. 1992;23:438.

24. Holden MK, Gill KM, Magliozzi MR, Nathan J, Piehl-Baker L. Clinical gait assessment in the neurologically impaired. Reliability meaningfulness Phys Ther. 1984;64:35-40. https://doi.org/10.1093/ptj/64.1.35.

25. Shah S, Vanclay F, Cooper B. Improving the sensitivity of the Barthel Index for stroke rehabilitation. J Clin Epidemiol. 1989;42:703-9. https://doi.org/https://doi.org/10.1016/0895-4356(89)90065-6.

26. Seripa D, D'Onofrio G, Panza F, Cascavilla L, Masullo C, Pilotto A. The genetics of the human APOE polymorphism. Rejuvenation Res. 2011;14:491-500. https://doi.org/10.1089/rej.2011.1169.

27. Dong Y, Hu B, Huang S, Ye T, Dong Q. The Modified Volume-Viscosity Swallow Test as a Predictor of Aspiration Pneumonia after Acute Ischemic Stroke. Clin Neurol Neurosurg. 2021;200:106351. https://doi.org/10.1016/j.clineuro.2020.106351.

28. Sekizawa K, Matsui T, Nakagawa T, Nakayama K, Sasaki H. ACE inhibitors and pneumonia. Lancet. 1998;352:1069. https://doi.org/10.1016/S0140-6736(05)60114-6.

29. Kumazawa R, Jo T, Matsui H, Fushimi K, Yasunaga H. Association between Angiotensin-Converting Enzyme Inhibitors and PostStroke Aspiration Pneumonia. J Stroke Cerebrovasc Dis. 2019;28:104444. https://doi.org/10.1016/j.jstrokecerebrovasdis.2019.104444.

30. Zhang H, Wu L-M, Wu J. Cross-Talk between Apolipoprotein E and Cytokines. Mediators Inflamm. 2011;2011:949072. https://doi.org/10.1155/2011/949072. 
31. Yao X, Gordon EM, Figueroa DM, Barochia AV, Levine SJ. Emerging Roles of Apolipoprotein E and Apolipoprotein A-I in the Pathogenesis and Treatment of Lung Disease. Am J Respir Cell Mol Biol. 2016;55:159-69. https://doi.org/10.1165/rcmb.20160060TR.

32. Lagging C, Lorentzen E, Stanne TM, Pedersen A, Soderholm M, Cole JW, Jood K, Lemmens R, Phuah CL, Rost NS, Thijs V, Woo D, Maguire JM, Lindgren A, Jern C, Genetics of Ischaemic Stroke Functional Outcome n, the International Stroke Genetics C (2019) APOE epsilon4 is associated with younger age at ischemic stroke onset but not with stroke outcome. Neurology 93:849-853. https://doi.org/10.1212/WNL.0000000000008459.

33. van der Lee SJ, Wolters FJ, Ikram MK, Hofman A, Ikram MA, Amin N, van Duijn CM. The effect of APOE and other common genetic variants on the onset of Alzheimer's disease and dementia: a community-based cohort study. Lancet Neurol. 2018;17:434-44. https://doi.org/https://doi.org/10.1016/S1474-4422(18)30053-X.

34. Robinson AC, Davidson YS, Roncaroli F, Minshull J, Tinkler P, Horan MA, Payton A, Pendleton N, Mann DMA. Influence of APOE Genotype on Mortality and Cognitive Impairment. Journal of Alzheimer's Disease Reports. 2020;4:281-6. https://doi.org/10.3233/ADR-200203.

35. Altamura C, Squitti R, Pasqualetti P, Tibuzzi F, Silvestrini M, Ventriglia MC, Cassetta E, Rossini PM, Vernieri F. What is the relationship among atherosclerosis markers, apolipoprotein E polymorphism and dementia? Eur J Neurol. 2007;14:679-82. https://doi.org/10.1111/j.1468-1331.2007.01714.x.

36. Gale SC, Gao L, Mikacenic C, Coyle SM, Rafaels N, Murray Dudenkov T, Madenspacher JH, Draper DW, Ge W, Aloor JJ, Azzam KM, Lai L, Blackshear PJ, Calvano SE, Barnes KC, Lowry SF, Corbett S, Wurfel MM, Fessler MB. APOع4 is associated with enhanced in vivo innate immune responses in human subjects. J Allergy Clin Immunol. 2014;134:127-34. https://doi.org/10.1016/j.jaci.2014.01.032.

37. Gerard HC, Fomicheva E, Whittum-Hudson JA, Hudson AP. Apolipoprotein E4 enhances attachment of Chlamydophila (Chlamydia) pneumoniae elementary bodies to host cells. Microb Pathog. 2008;44:279-85. https://doi.org/10.1016/j.micpath.2007.10.002.

38. Kuo C-L, Pilling LC, Atkins JL, Masoli JAH, Delgado J, Kuchel GA, Melzer D. APOE e4 Genotype Predicts Severe COVID-19 in the UK Biobank Community Cohort. The Journals of Gerontology: Series A. 2020;75:2231-2. https://doi.org/10.1093/gerona/glaa131.

39. Galovic M, Leisi N, Pastore-Wapp M, Zbinden M, Vos SB, Mueller M, Weber J, Brugger F, Kagi G, Weder BJ. Diverging lesion and connectivity patterns influence early and late swallowing recovery after hemispheric stroke. Hum Brain Mapp. 2017;38:2165-76. https://doi.org/10.1002/hbm.23511.

40. Galovic M, Stauber AJ, Leisi N, Krammer W, Brugger F, Vehoff J, Balcerak P, Muller A, Muller M, Rosenfeld J, Polymeris A, Thilemann S, De Marchis GM, Niemann T, Leifke M, Lyrer P, Saladin P, Kahles T, Nedeltchev K, Sarikaya H, Jung S, Fischer U, Manno C, Cereda CW, Sander JW, Tettenborn B, Weder BJ, Stoeckli SJ, Arnold M, Kagi G. Development and Validation of a Prognostic Model of Swallowing Recovery and Enteral Tube Feeding After Ischemic Stroke. JAMA Neurol. 2019;76:561-70. https://doi.org/10.1001/jamaneurol.2018.4858.

41. Suntrup S, Kemmling A, Warnecke T, Hamacher C, Oelenberg S, Niederstadt T, Heindel W, Wiendl H, Dziewas R. The impact of lesion location on dysphagia incidence, pattern and complications in acute stroke. Part 1: dysphagia incidence, severity and aspiration. Eur J Neurol. 2015;22:832-8. https://doi.org/10.1111/ene.12670.

42. Chang WH, Bang OY, Shin YI, Lee A, Pascual-Leone A, Kim YH. BDNF polymorphism and differential rTMS effects on motor recovery of stroke patients. Brain Stimul. 2014;7:553-8. https://doi.org/10.1016/j.brs.2014.03.008.

\section{Figures}


APoE $\varepsilon 4(-)$

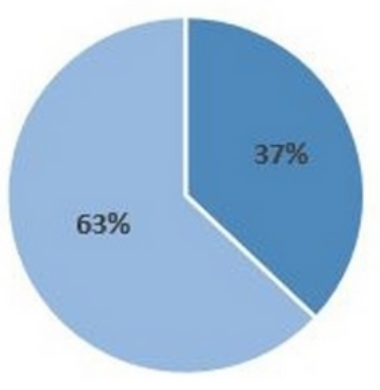

APoE $\varepsilon 4(-)$

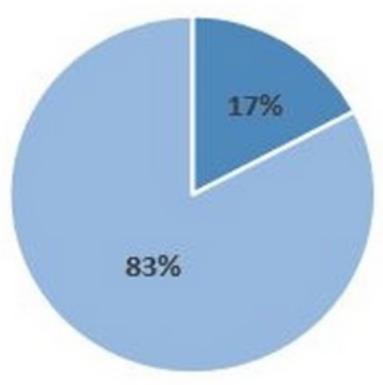

APoE $\varepsilon 4(-)$

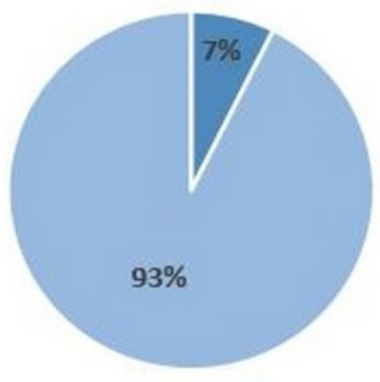

APoE $\varepsilon 4(+)$

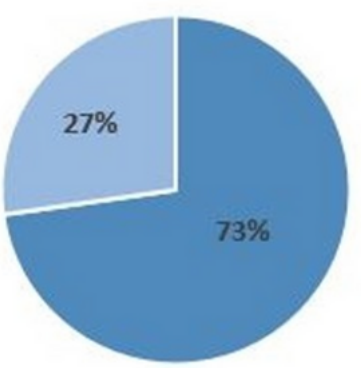

APoE $\varepsilon 4(+)$

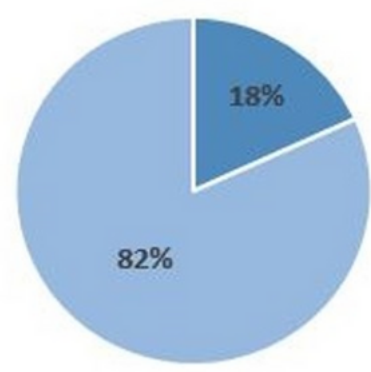

APoE $\varepsilon 4(+)$

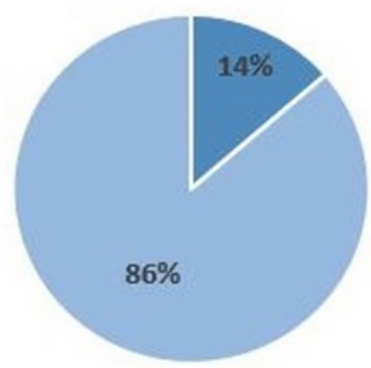

\section{Figure 1}

Association of infection and APOE genotypes in the young age group. The incidence of post-stroke aspiration pneumonia was significantly higher in those with $A P O E \otimes 4(P=0.003)$. The incidence of urinary tract infections $(P=0.922)$ and colitis $(P=0.359)$ did not differ according to APOE genotypes.

\section{Supplementary Files}

This is a list of supplementary files associated with this preprint. Click to download.

- Supplementary.docx 